\title{
Combining lon Mobility and Cryogenic Spectroscopy for Structural and Analytical Studies of Biomolecular Ions
}

\author{
Michael Z. Kamrath ${ }^{\dagger}$ and Thomas R. Rizzo*(1) \\ Laboratoire de Chimie Physique Moléculaire, École Polytechnique Fédérale de Lausanne, EPFL SB ISIC LCPM, Station 6, CH-1015 \\ Lausanne, Switzerland
}

CONSPECTUS: Ion mobility spectrometry (IMS) has become a valuable tool in biophysical and bioanalytical chemistry because of its ability to separate and characterize the structure of gas-phase biomolecular ions on the basis of their collisional cross section (CCS). Its importance has grown with the realization that in many cases, biomolecular ions retain important structural characteristics when produced in the gas phase by electrospray ionization (ESI). While a CCS can help distinguish between structures of radically different types, one cannot expect a single number to differentiate similar conformations of a complex molecule. Molecular spectroscopy has also played an increasingly important role for structural characterization of biomolecular ions. Spectroscopic measurements, particularly when performed at cryogenic temperatures, can be extremely sensitive to small changes in a molecule's conformation and provide tight constraints for calculations of biomolecular structures. However, spectra of complex molecules can be heavily congested due to the presence of multiple stable conformations, each of which can have a distinct spectrum. This congestion can inhibit spectral analysis and complicate the extraction of structural information. Even when a single conformation is present, the conformational search process needed to match a measured spectrum with a computed structure can be overwhelming for peptides of more than a few amino acids, for example.

We have recently combined ion mobility spectrometry and cryogenic ion spectroscopy (CIS) to characterize the structures of gas-phase biomolecular ions. In this Account, we illustrate how the coupling of IMS and CIS is by nature synergistic. On the one hand, IMS can be used as a conformational filter to reduce spectral congestion that arises from heterogeneous samples, facilitating structural analysis. On the other hand, highly resolved, cryogenic spectra can serve as a selective detector for IMS that can increase the effective resolution and hence the maximum number of distinct species that can be detected. Taken together, spectra and CCS measurements on the same system facilitates structural analysis and strengthens the conclusions that can be drawn from each type of data.

After describing different approaches to combining these two techniques in such a way as to simplify the data obtained from each one separately, we present two examples that illustrate the type of insight gained from using spectra and CCS data together for characterizing gas-phase biomolecular ions. In one example, the CCS is used as a constraint for quantum chemical structure calculations of kinetically trapped species, where a lowest-energy criterion is not applicable. In a second example, we use both the CCS and a cryogenic infrared spectrum as a means to distinguish isomeric glycans.

\section{INTRODUCTION}

The use of ion mobility spectrometry (IMS) has grown considerably in recent years as a biophysical/bioanalytical tool. ${ }^{1}$ It has found applications in structural biology, where the rotationally averaged collision cross section (CCS) provides a qualitative picture of the structure adopted by a large biomolecule. ${ }^{2,3}$ If the molecule or complex is not too large, high level computations can help determine the structure more precisely. In this case, the CCS determined experimentally can be used to validate certain calculated structures from a larger pool of potential conformations. ${ }^{4}$

Another important and complementary technique used to characterize the structure of biomolecular ions is gas-phase spectroscopy. ${ }^{5-7}$ Spectroscopy provides a characteristic fingerprint of a molecule that is highly sensitive to its structure, particularly when performed at cryogenic temperatures. In the case of IR spectroscopy, for example, one obtains a vibrational signature, which can be used in conjunction with quantum chemical calculations to assign three-dimensional structures.

While both IMS and cryogenic ion spectroscopy provide valuable information on their own, the power of each of these techniques can be increased by a combination of the two. One way to see this is to imagine a three-dimensional surface in which one plots the signal intensity versus drift time along one axis and wavenumber along an orthogonal axis. One can exploit the synergy of these two techniques either by taking cuts along such a surface to simplify the data in one dimension or the other or by using the combined information from the entire surface to more fully characterize a complex biomolecular ion.

In this Account, we first use a few examples from our laboratory to illustrate different ways that such a hypothetical surface could be cut and demonstrate how such cuts can

Received: March 22, 2018

Published: May 10, 2018 
provide additional resolution. We then go on to describe two examples where combining spectroscopic information with ion mobility can be used for either structural analysis or analytical studies of biomolecular ions.

\section{ION MOBILITY AS A CONFORMATIONAL FILTER FOR CRYOGENIC ION SPECTROSCOPY}

As molecules become larger and more complex, their spectra become increasingly congested. In gas-phase experiments, this congestion often arises from conformational heterogeneity, where distinct spectra of multiple conformers overlap, making it difficult to disentangle them and extract detailed structural information. If these conformers are sufficiently distinct, one could envision using ion mobility to separate them prior to spectroscopic investigation, reducing spectral congestion and simplifying spectral assignment. Employing ion mobility as a conformational filter would be the equivalent of taking a cut along a plane of the hypothetical multidimensional surface mentioned above. This would allow one to isolate the spectrum of the transmitted conformer(s).

Three conditions must be met for this basic strategy to work: (1) ion mobility must be able to resolve conformers or conformational families for a given molecule, (2) the isolated conformers must be inherently stable on the time scale of the experiment, and (3) conformational interconversion induced by energizing the ions after mobility separation and prior to spectroscopic analysis must be avoided. Clemmer and coworkers first demonstrated that requirements 1 and 2 could be met for ubiquitin $[\mathrm{M}+7 \mathrm{H}]^{7+}$ in a helium-filled linear drift tube ${ }^{8}$ and later showed similar results for bradykinin $[\mathrm{M}+$ $3 \mathrm{H}]^{3+}$. . Meeting requirement 3 is largely a question of managing the collision energies in the experiment, and Vonderach et al. demonstrated the feasibility of this approach by recording distinct photoelectron spectra for four mobilityseparated conformers of the all-cytosine DNA hexanucleotide. $^{10}$

We have recently constructed an instrument, shown schematically in Figure 1, that combines drift-tube ion mobility



Figure 1. Apparatus combining drift-tube ion mobility and cryogenic spectroscopy. Adapted with permission from ref 12. Copyright 2017 Springer.

with cryogenic IR spectroscopy in which the former can be used as a conformational filter for the latter., ${ }^{41}$ The drift tube is of the Clemmer design, ${ }^{9}$ which incorporates an ion funnel halfway down the axis of the tube, together with a selection gate and activation zone. The selection gate can be used to slice out a packet of ions with a particular drift time in the first segment of the tube, allowing one to perform IMS-IMS experiments.
The resolution of the second half of the drift tube after slicing is $\sim 55$. One can also collisionally energize the selected ions in the activation zone before measuring their drift time in the second half of the tube.

Once exiting the drift tube, the ions pass through a quadrupole mass filter and are guided into a planar, cryogenic ion trap. ${ }^{11}$ The trap is made from a pair of PC boards onto which a series of RF and DC electrodes are printed. The boards are in contact with a copper housing, which is in contact with a closed-cycle helium cryostat maintained at $\sim 13 \mathrm{~K}$, into which helium is pulsed. As the packet of mass- and mobility-selected ions arrive in the trap, they are cooled through collisions with a $\mathrm{He} / \mathrm{H}_{2}$ buffer gas mixture and "tagged" with one or more $\mathrm{H}_{2}$ molecules. ${ }^{13}$ Since the binding energy of $\mathrm{H}_{2}$ is small $(<500$ $\mathrm{cm}^{-1}$ ), it generally has only a slight effect on the structure of the parent molecule and can easily be dissociated with a single infrared photon. ${ }^{14}$ This allows us to use the $\mathrm{H}_{2}$ as a mass messenger when performing infrared spectroscopy of the cold ions. To do this, we irradiate the ions with an IR laser pulse either directly in the trap or shortly after we eject them through slots milled between the RF electrodes. We monitor the depletion of the $\mathrm{H}_{2}$ adducts in a reflectron time-of-flight mass spectrometer as a function of laser wavenumber to generate an infrared spectrum.

As an example of how ion mobility can be used as a conformational filter for cryogenic ion spectroscopy, we show in Figure 2 the drift time distribution (converted to CCS) and vibrational spectrum of two conformational families of the $\mathrm{N}$ terminal 1-5 fragment of the peptide bradykinin. ${ }^{4}$ One can see that the vibrational spectrum of each conformational family is almost completely separated from the other; there are few, if any, spectral features of one conformation in the spectrum of the other. The ability to separate the vibrational spectra into contributions from different conformers or conformer families greatly simplifies structure determination by comparison with computed spectra.

We have used a similar approach in our study of GPGG ${ }^{11}$ and cabTRP Ia, ${ }^{15}$ and we discuss below how the combined information from the drift-time distribution and the vibrational spectra greatly helps in determining the three-dimensional structure of the ion.

We have also shown that the same principle can be employed using other types of ion mobility separation, such as field asymmetric ion mobility spectrometry (FAIMS). ${ }^{16}$ FAIMS separates ions on the basis of their difference in mobility through a carrier gas at high and low electric field. ${ }^{17-19}$ In FAIMS, ions travel between two electrodes to which an asymmetric, alternating waveform is applied consisting of a high field component of one polarity followed by a low field component of the opposite polarity. This waveform induces ion motion perpendicular to that of the carrier gas, causing the ions to drift toward one of the electrodes or the other, depending upon the difference in its mobility at high and low fields. If left uncorrected, these ions would be lost upon colliding with one of the electrodes, but they can be transmitted through the FAIMS device if a DC compensation voltage $(\mathrm{CV})$ is applied to correct the ion trajectory. A FAIMS "spectrum" is obtained by monitoring the ion transmission through the device as a function of the CV. Our instrument, part of which is shown schematically in Figure 3, combines a transverse cylindrical geometry FAIMS source as described previously. ${ }^{17-19}$ To measure a spectrum of mobility-selected ions, we set the CV at an appropriate value to transmit those corresponding to a 



Figure 2. Ion mobility data (left panel) and cryogenic vibrational spectra of individual conformer families of bradykinin $[1-5][\mathrm{M}+2 \mathrm{H}]^{2+}($ right panel) measured by messenger-tagging spectroscopy. ${ }^{4}$

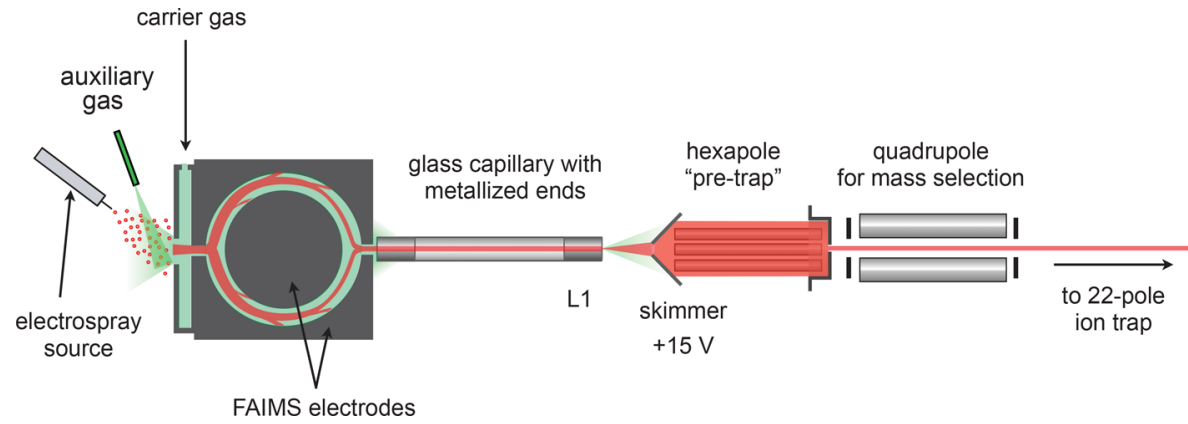

Figure 3. Schematic showing the coupling of our FAIMS source to a cryogenic 22-pole ion trap. Reproduced with permission from ref 20 . Copyright 2012 Springer.
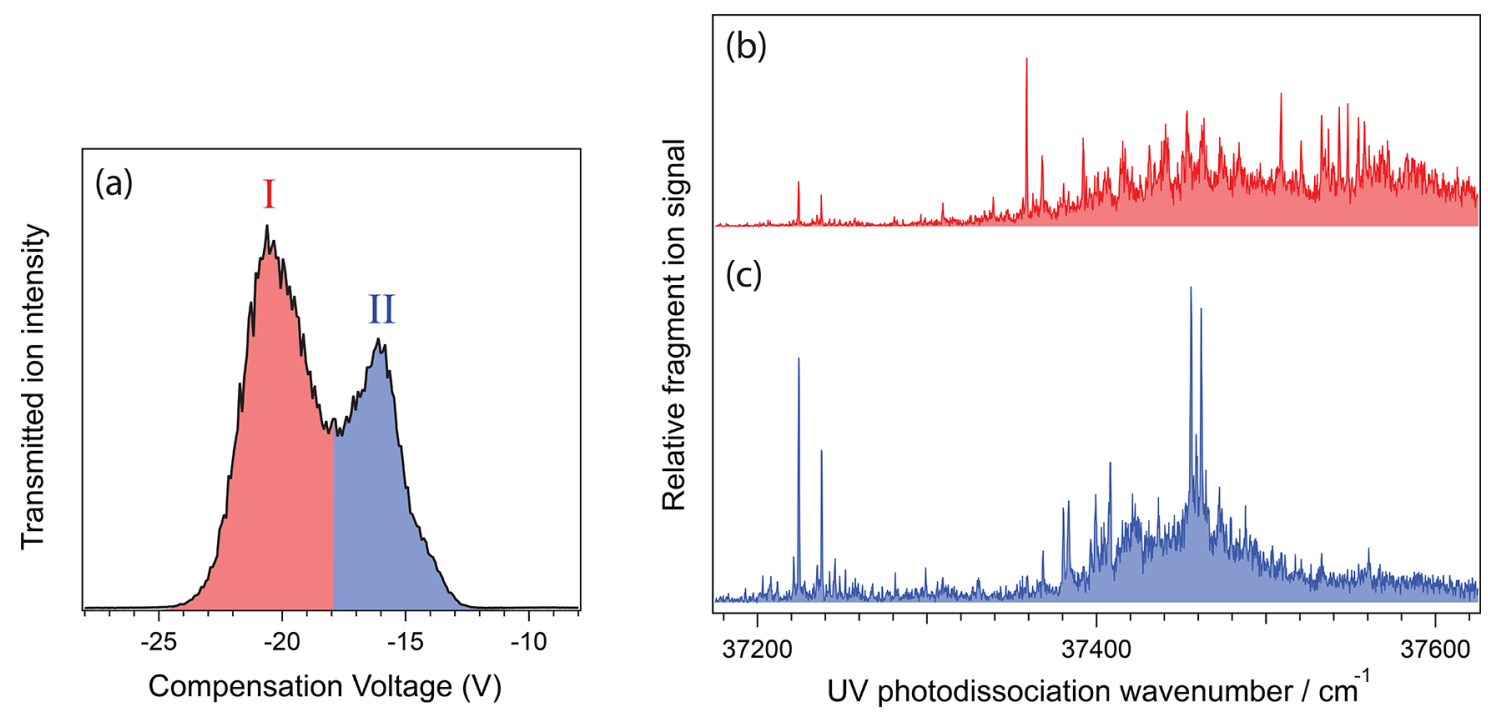

Figure 4. FAIMS CV spectrum (a) and electronic spectra (b, c) of bradykinin $[\mathrm{M}+2 \mathrm{H}]^{2+} \cdot{ }^{20}$ The spectrum of conformational family I (a) was measured at a compensation voltage of $-20.4 \mathrm{~V}$, while that of family II (b) was measured at a CV of $-16.1 \mathrm{~V}$. Adapted with permission from ref 19 . Copyright 2012 Springer.

particular conformer, collect them in a hexapole trap, and then pass them through a quadrupole mass filter before sending them to a cryogenic 22-pole ion trap. ${ }^{20}$ We then interrogate them with a tunable UV dye laser and detect photon absorption by monitoring photoinduced fragmentation using a second quadrupole mass filter. Measuring the intensity of the photofragment ion signal as a function of laser wavelength generates a UV spectrum of the cold ions.

We have used the device of Figure 3 to separate the electronic spectrum of the $[\mathrm{M}+2 \mathrm{H}]^{2+}$ state of cryogenically cooled bradykinin into contributions from different conformational families. ${ }^{16,20}$ Bradykinin is a 9-amino acid peptide known to have a high degree of backbone flexibility. ${ }^{21}$ Its UV spectrum is highly congested, even at low temperatures, largely because of the superposition of spectra of at least five different stable conformers. $^{22}$ The CV spectrum of bradykinin $[\mathrm{M}+2 \mathrm{H}]^{2+}$, shown in Figure $4 \mathrm{a}$, clearly shows two conformational families that are partially resolved. By setting the compensation voltage at the peak of either of these conformational families, one can measure the electronic spectrum of the selectively transmitted 

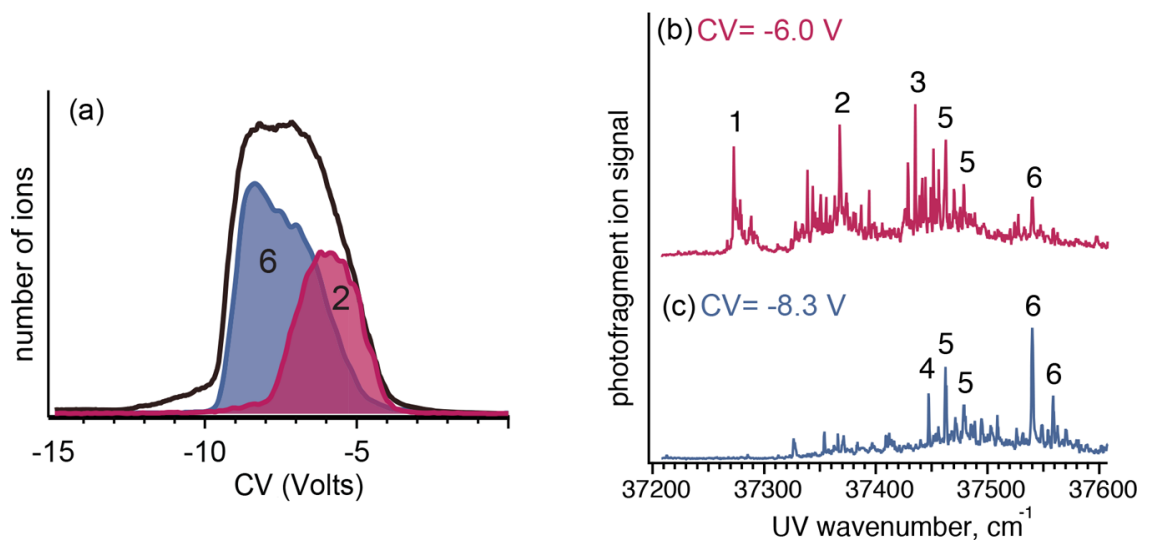

Figure 5. (a) CV spectrum of the Pro3Ala and Pro7Ala mutant of $\mathrm{BK}^{3+}$. The black trace represents the distribution monitoring the total transmitted ion current, while the blue and pink traces were measured via UV transitions attributed to conformers 6 and 2, respectively. (b) UV photofragment spectrum of the Pro3Ala and Pro7Ala mutant of $\mathrm{BK}^{3+}$ with the compensation voltage set to $-6.0 \mathrm{~V}$; (c) same as panel b but with the compensation voltage set to $-8.3 \mathrm{~V}$.

ions, shown in Figure 4b,c. A clear difference can be seen between the UV photofragmentation spectra of these two conformational families, demonstrating that FAIMS can be effective as a conformational filter for spectroscopy, even though the resolution of the FAIMS device is rather modest $(\sim 6)$.

The separation is not $100 \%$, however; one can see a small amount of conformer family II in the spectrum of family I, particularly the two strong peaks between 37210 and 37220 $\mathrm{cm}^{-1}$, although there seems to be no contamination in the other direction. This implies that the contamination comes from interconversion among conformers after FAIMS separation rather than insufficient FAIMS resolution. This is likely caused by ions experiencing collisions with energies of the same order as the barriers that separate the different conformational states and could present a fundamental limit when using any type of ion mobility as a filter for spectroscopy. To minimize high-energy collisions, one must use low acceleration voltages in regions of intermediate pressure.

There are several other groups that have applied the general approach of using ion mobility as a conformational filter for spectroscopy. For example, Zenobi and co-workers used an atmospheric-pressure differential-mobility analyzer to isolate ions before characterizing their structures with laser-induced fluorescence. $^{23,24}$ Von Helden and co-workers combined drifttube ion mobility with room-temperature IR multiphoton dissociation to assign the structures of benzocaine. ${ }^{25}$ Recent work from Daly et al. has demonstrated the use of drift-tube ion mobility in combination with fluorescence resonance energy transfer to monitor the collision-induced unfolding of the protein ubiquitin. $^{26}$ Together with our own work, these examples demonstrate the creative ways that ion mobility can be used as a spectroscopic filter for characterizing complex biomolecular structures in the gas phase.

\section{SPECTROSCOPY AS A SELECTIVE DETECTOR FOR ION MOBILITY}

As described above, using ion mobility as a filter for cryogenic ion spectroscopy increases one's ability to distinguish spectroscopic features by removing or reducing conformational heterogeneity, facilitating spectroscopic analysis. However, conformational heterogeneity also poses a problem for ion mobility. Current ion mobility techniques have sufficient resolution to detect major structural differences between biomolecular ions, particularly when they correspond to different backbone configurations, but they often have difficulty detecting more subtle structural variations. Within an IMS arrival time distribution, there are likely to be multiple stable conformations of a molecule that are difficult to resolve. Cryogenic spectroscopy, on the other hand, is exquisitely sensitive to even the smallest of structural differences, since one can detect minute shifts in vibrational or electronic transition frequencies. If, rather than measuring ion mobility by detecting total ion current, one detects only those ions that absorb a photon of a particular frequency, one could reduce or even eliminate the remaining conformational heterogeneity by isolating the contribution of individual components to the IMS distribution. The net effect would be to increase the ability of ion mobility to distinguish extremely subtle structural differences.

In principle, such an approach is applicable to different types of ion mobility (i.e., drift-tube, traveling-wave, FAIMS, or trapped-ion mobility spectrometry (TIMS)), different types of spectroscopy (i.e., vibrational, electronic), and different methods of spectroscopic detection (i.e., photofragmentation, messenger tagging, laser-induced unfolding, or infrared multiple photon dissociation (IRMPD)). To demonstrate the principle, we have implemented this approach by combining FAIMS with UV photofragment spectroscopy. Figure 5a shows a CV spectrum of a mutant of the triply protonated peptide bradykinin in which the prolines in positions 3 and 7 have been substituted by alanine. ${ }^{27}$ The FAIMS CV distribution detecting the total ion current (black trace) is unresolved, but its shape clearly implies the presence of several different species. Figure $5 b, c$ shows UV photofragment spectra at fixed values of the $\mathrm{CV}$, using FAIMS as a conformational filter for the electronic spectrum. The labels on the spectroscopic features denote different conformations from which they arise, which have been confirmed by IR-UV double resonance. ${ }^{27}$

Having identified spectroscopic features that are unique to different conformers, we can use these to detect the ions transmitted by FAIMS in a conformation-specific manner. The blue and pink traces in Figure 5a show the CV spectrum of those ions that absorb UV radiation at features corresponding to conformers 2 and 6 . In doing so, we decompose the CV spectrum into components from individual conformers. While 
we have demonstrated this approach using FAIMS and UV photofragment spectroscopy, it should be perfectly amenable to any type of ion mobility and with a variety of different types of spectroscopy.

Clearly, some spectroscopic knowledge is needed to perform the decomposition process, which in the case described above has been done by UV photofragment spectroscopy (Figure $5 b, c)$. However, one does not need a fully assigned spectrum to decompose the ion mobility data. One simply needs a single spectroscopic feature that is unique to a specific conformer or conformational family. Additional spectroscopic data may nevertheless be helpful for interpreting the data.

\section{ION MOBILITY AS A SPECTROSCOPIC DETECTION METHOD}

To complete the discussion of different combinations of ion mobility and molecular spectroscopy, it is important to mention the use of ion mobility as a spectroscopic detection method. In this case, one uses the change in mobility subsequent to photon absorption as a means of spectroscopic detection. The cleanest way to do this would be to use an IMS-laser-IMS arrangement, in which one separates ions in the first IMS stage, intersects those ions with a laser, and then detects any photoinduced isomerization in the second IMS stage. The basic requirement for this to work is that an ion's collision cross section must change upon photon absorption in a measurable way.

Bieske and co-workers have constructed such an instrument using UV excitation in an IMS-UV-IMS arrangement and have tested the approach with the carbocyanine molecule $\mathrm{DTC}^{+}$, which is a system known to undergo cis/trans $\mathrm{C}-\mathrm{C}$ bond isomerization. ${ }^{28,29}$ When all ions traverse the drift tube without mobility selection or laser interaction, they observe two peaks in the mobility distribution, as shown in Figure 6. To switch to spectroscopy mode, they select either peak 1 or 2 (as shown in traces b and c, respectively) in the first IMS stage, before intersecting the ions with $532 \mathrm{~nm}$ light. The photoinduced response is then recorded, and the ratio is displayed by the red traces. Clearly, the photoexcitation leads to isomerization, as evidenced by a change in the drift-time distribution. They have extended the single wavelength measurement by scanning the laser, demonstrating that one can use this approach to obtain a UV spectrum of mobility-selected species. $^{29}$ Dugourd and co-workers report analogous results when exciting an opto-switchable peptide with a tunable UV/ vis OPO system using a similar experimental arrangement. ${ }^{30,31}$ Clearly, UV photoswitchable molecules are good targets for such an approach, since their collision cross sections tend to change considerably upon photon absorption. However, one could envision applying this method to record a vibrational spectrum, as long as the absorption of one or more infrared photons leads to a measurable change in the collision cross section.

\section{USING ION MOBILITY TO RESTRICT CONFORMATIONAL SEARCH SPACE IN INFRARED SPECTROSCOPIC STUDIES}

As the use of infrared spectroscopy for the determination of the structures of biomolecular ions has moved to increasingly larger and more complex systems, certain limitations arise that are difficult to overcome. From the experimental side, larger systems often have multiple stable conformations with

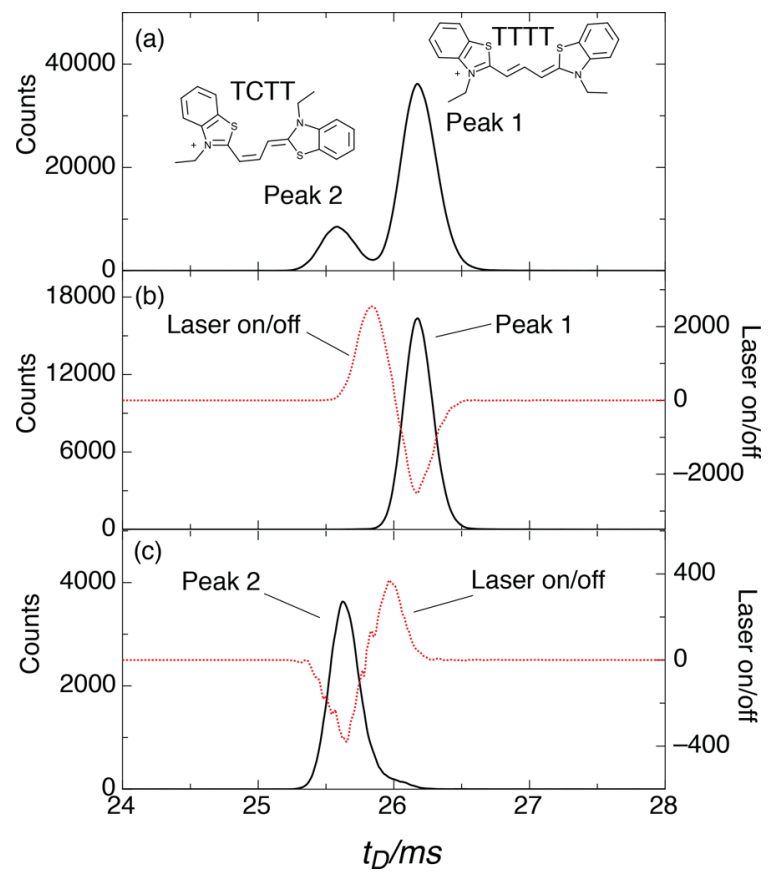

Figure 6. Drift-time distribution of (a) the carbocyanine, $\mathrm{DTC}^{+}$, without mobility selection or laser excitation. In panel $b$, peak 1 is mobility-selected and then interrogated with $532 \mathrm{~nm}$ wavelength light. The resulting ratio of laser on/off is shown by the red trace. Panel c shows the results of a similar experiment, when peak 2 is mobility selected. Reproduced with permission from ref 28. Copyright 2014 AIP Publishing.

overlapping spectra. To help mitigate this problem, one can use ion mobility as a conformational filter, as described above, or apply IR-UV double resonance techniques, or even combine the two. ${ }^{27}$ Nevertheless, even if one has a high-resolution, conformer-specific IR spectrum, extracting the structure from such a spectrum still poses a difficult challenge. The usual procedure is to compute the lowest-energy structures, calculate the spectrum for each of these structures, and then compare them with the measured spectrum. When a sufficiently good match is found between the measured and computed spectra, one assigns the computed structure to the molecule under investigation. However, as the size of the molecule increases, the computational effort to explore the conformational space can become formidable. One typically performs a fast calculation using force fields to determine the lowest energy structures, and then optimizes these structures using DFT. Unfortunately, the relative energy ordering of the states often changes substantially upon refinement with DFT, and thus one has still to perform quantum calculations for a considerable number of geometries, which simply may become prohibitive for larger molecules. This is where the interplay with ion mobility data can help. By using collisional cross sections derived from ion mobility, one already has a structural constraint for calculated geometries that can reduce the conformational search space and hence the number of geometries that need to be optimized via DFT. Even if the CCS is not used explicitly to reduce the search space, it nevertheless serves as an additional constraint to impose on calculated geometries. ${ }^{11,32}$

The problem of assigning a molecular geometry based on the comparison of computed infrared spectra with those from experiment becomes particularly acute when one cannot rely on 
a lowest energy criterion, and this occurs in cases where one encounters kinetically trapped structures. When producing biomolecular ions directly from solution using electrospray, it is clear that the potential energy surface, and hence the geometry of the lowest energy structures, will change as one removes increasing amounts of solvent, as illustrated schematically in Figure 7a. However, if the barriers to conformational
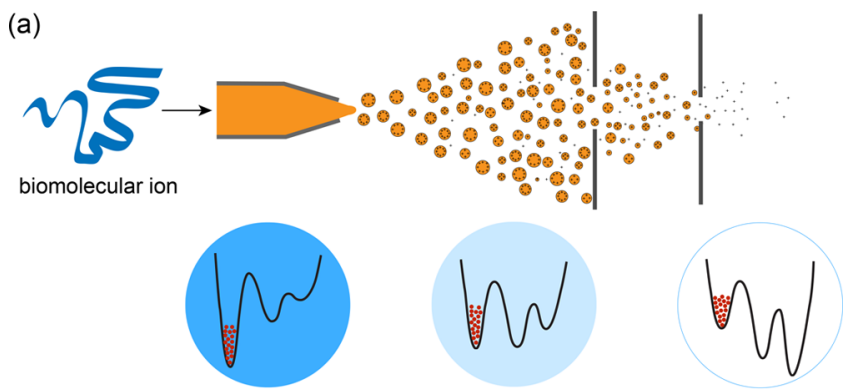

(b)

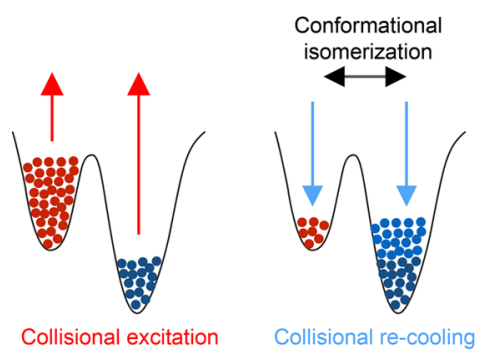

Figure 7. Schematic showing the process of (a) kinetic trapping of electrosprayed ions and (b) annealing of a kinetically trapped conformer distribution.

isomerization are high relative to thermal energies, "solutionlike" structures may be trapped in the gas phase. One piece of evidence for kinetic trapping is that upon collisional heating in the gas phase, metastable conformations can be induced to isomerize, and when collisionally recooled, they exhibit a conformer distribution that is different from the original. ${ }^{8,33-36}$ This annealing process is shown schematically in Figure $7 \mathrm{~b}$.

A variety of gas-phase techniques have been used to investigate kinetically trapped species, since they provide the opportunity to obtain insight on structures that retain some "memory" of their conformation in solution. ${ }^{35,37-40}$ While the use of vibrational spectroscopy is one of the most powerful gasphase approaches for structure determination, the theoretical treatment needed to go from the measured spectrum to a molecular geometry is particularly problematic in this case. This is because by definition, kinetically trapped species are not the lowest energy structures, and in many cases, one has no idea of their energy relative to the most stable gas-phase species. This means that one cannot rely upon energy minimization to find them in a conformational search. In this case, the use of ion mobility data together with vibrational spectra can be particularly helpful insofar as the collisional cross section provides a conformational constraint.

To illustrate this, we return to our study of the N-terminal fragment of bradykinin, which has the sequence Arg-Pro-ProGly-Phe. ${ }^{4}$ The two proline residues are known to introduce conformational heterogeneity via cis/trans isomerization. In our investigation, we first probed the system in solution using NMR spectroscopy and determined that the fragment overwhelm- ingly adopts a trans-Pro ${ }^{2} /$ trans-Pro ${ }^{3}$ conformation. We then used ion mobility to record the drift-time distribution shown in the bottom panel of Figure 8, which primarily consists of features at 167 and $178 \AA^{2}$.

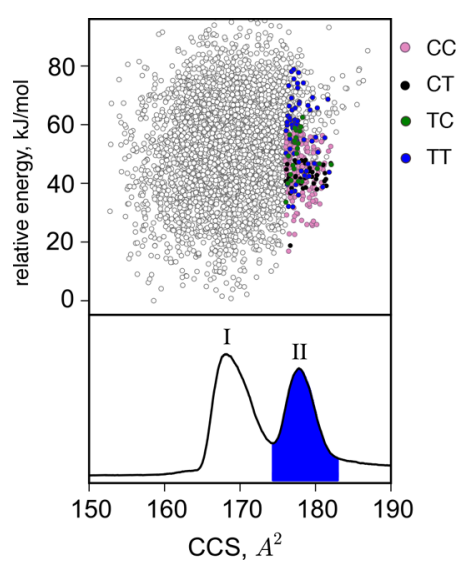

Figure 8. Distribution of relative potential energies of considered conformers as a function of CCS. Those conformations for which the vibrational spectra were computed are shown in color. The rest of the structures are shown in gray.

Upon annealing this conformer distribution, the more extended structure at $178 \AA^{2}$ converts almost entirely to the more compact one, indicating that the former is kinetically trapped. Because the compact conformer appears to be the lowest energy gas-phase structure, we analyzed it using cryogenic vibrational spectroscopy, together with "standard" conformational search methods, first using force fields to identify the lowest energy structures and then optimizing them by DFT. We thus determined that the more compact species consists of two populations of ions: cis-Pro ${ }^{2} /$ trans-Pro ${ }^{3}$ and trans-Pro $2 /$ cis-Pro ${ }^{3}$. For analysis of the kinetically trapped species with a CCS of $178 \AA^{2}$, however, one cannot rely on the energy criterion, since by definition, kinetically trapped species are higher in energy and separated from the lowest energy structures by substantial barriers on the potential energy surface. In this case, we used the CCS as a cutoff, only considering structures higher $176 \AA^{2}$, as shown in Figure 8, and for these we used a relatively high energy threshold. Among these candidates, we then selected the structure that best accounted for the spectroscopic observables. This analysis revealed that both proline residues adopt a trans-type geometry. This is consistent with what we observed in solution and implies that the solution-preferred structure retains its backbone geometry when produced in the gas phase by electrospray.

\section{ION MOBILITY AND SPECTROSCOPY AS AN ANALYTICAL TOOL FOR IDENTIFICATION}

The combined information from ion mobility and vibrational spectroscopy not only is useful for structural studies of gasphase biological molecules but also could be used for analytical purposes. In characterizing a heterogeneous sample, one must often resort to multidimensional analysis using so-called "hyphenated" techniques whenever a single analytical method is insufficient to resolve or characterize the individual components. Common combinations are liquid chromatography with mass spectrometry (LC-MS) and ion mobility with mass spectrometry (IMS-MS). For certain difficult problems, 


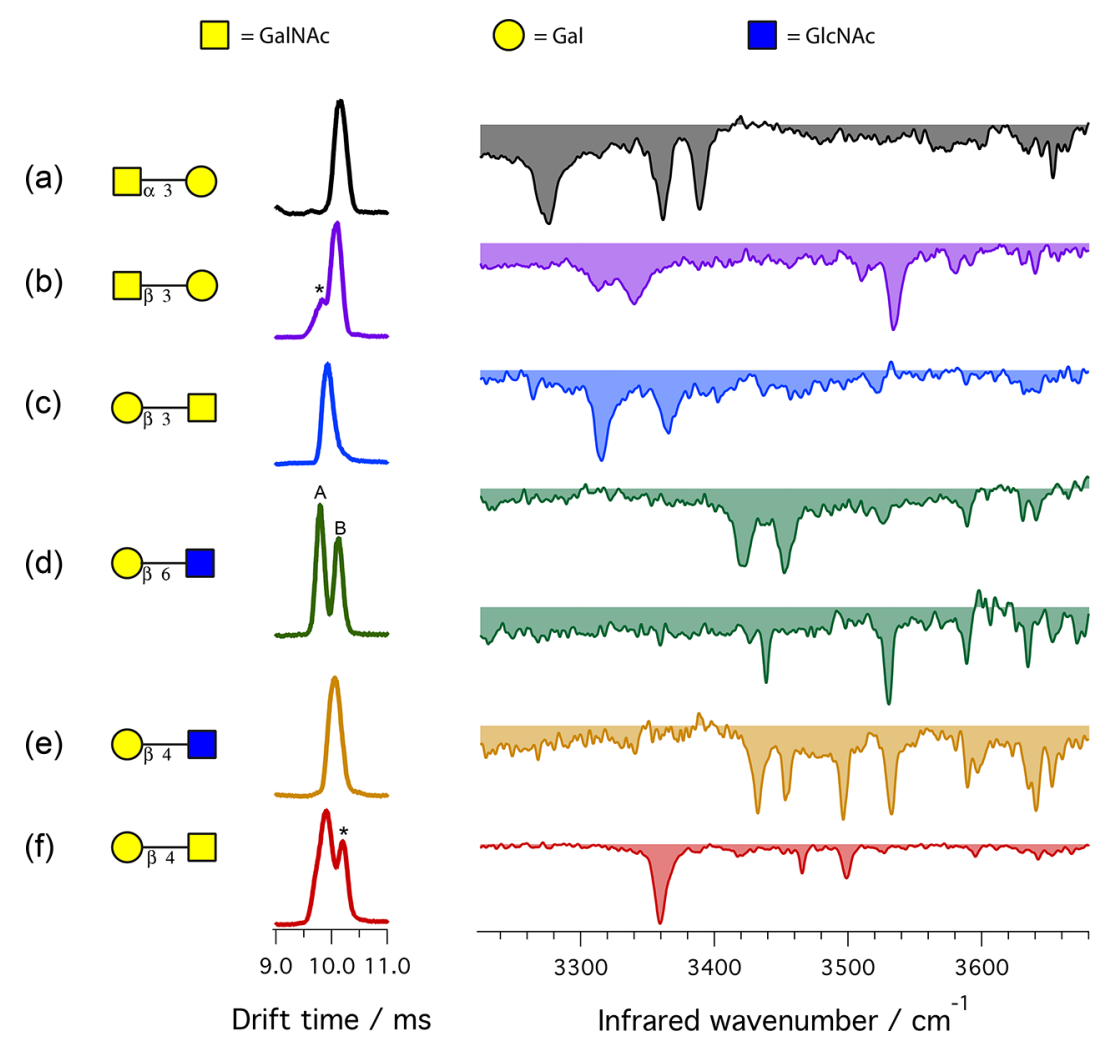

Figure 9. IMS arrival-time distributions and fingerprint vibrational spectra for six isomeric disaccharides that differ in their anomericity (a vs b), sequence order ( $b$ vs c), attachment point ( $d$ vs e), and monosaccharide content (e vs $f$ ). The two vibrational spectra in panel d correspond to two stable conformations that do not interconvert upon annealing. The asterisk on the arrival time distributions of panels $\mathrm{b}$ and $\mathrm{f}$ come from a species of slightly different mass that was passed by the selecting quadrupole. Reproduced with permission from ref 12 . Copyright 2017 Springer.

adding a third dimension can be useful and even necessary. One such problem arises in the identification of oligosaccharides, or glycans, because of their inherent isotopic complexity. The isomeric nature of the monosaccharide building blocks, the stereochemistry of the glycosidic bond, the possibility of multiple attachment points, and the occurrence of isomeric branched structures all make glycans particularly difficult to analyze. Mass spectrometry alone cannot distinguish isomeric species, and while IMS can resolve certain different types of isomers, it cannot distinguish the subtlest differences between stereoisomers. On the other hand, cryogenic vibrational spectroscopy is exquisitely sensitive to the slightest differences in molecular structure and can easily distinguish isomers.

We have recently combined IMS, cryogenic ion spectroscopy, and MS for glycan identification, using the apparatus of Figure $1 .{ }^{12}$ Figure 9 shows our measurement of the drift-time distributions and cryogenic vibrational spectra on a series of six sodiated isomeric disaccharides. While the former largely overlap, it is clear that the vibrational fingerprint spectra are completely distinct. For example, the spectrum in part $\mathrm{f}$ displays a strong peak at $3360 \mathrm{~cm}^{-1}$, which is completely absent in part e. This may seem remarkable considering that the only difference between the two isomers is the orientation of a hydroxyl group about a single stereogenic carbon. However, such spectral differences are typical in the cryogenic vibrational analysis of glycans due to the strongly coupled nature of the $\mathrm{OH}$ stretch bands.

One could use ion mobility and spectroscopic data to identify glycans by constructing a database that tabulates the mass, CCS, and vibrational spectrum of glycan standards. In the case of a single component sample, one would simply measure these quantities and then identify a match from the database. For a multicomponent sample, however, one may have species with the same mass and CCS, and the vibrational information one collects would be a superposition of these overlapping species. Nevertheless, one could decompose such a vibrational spectrum into a sum of spectra from the database and identify the contributing compounds.

One should note that we collected the data of Figure 9 using the sodiated rather than the protonated glycan species. While complexation with sodium may promote more compact structures, thereby minimizing CCS differences in certain classes of glycans, other isomers, particularly ones exhibiting chain branching, remain distinct in both their CCS and the vibrational spectrum, even when sodiated. ${ }^{12}$

While we highlight the utility of ion mobility and spectroscopy to aid in targeted glycan identification, these results portend successful application of this approach to other isomer- or conformer-rich systems. In such cases, this technique could provide a suitable alternative to the more common HPLC- or GC-based approaches to molecular identification.

\section{CONCLUSIONS AND OUTLOOK}

In this Account, we have tried to demonstrate that the combination of ion mobility and spectroscopy is synergistic and provides powerful new ways to characterize gas-phase biomolecular ions. From an experimental point of view, each technique can be used to simplify data from the other by removing conformational heterogeneity. Ion mobility can act as a conformational filter, thereby reducing spectral congestion, and spectroscopy can allow one to pull apart highly congested IMS spectra using unique absorptions of each species. We have 
also tried to show how the information from these two techniques can be applied together. The use of collision cross sections measured by ion mobility can reduce the conformational search space for quantum calculations of the molecular structure, and in the case of kinetically trapped species, it provides experimental constraints when the lowest-energy criterion is not valid. We have also shown that the combination of IMS data with cryogenic vibrational spectroscopy can be used to identify isomeric glycans, which are difficult to characterize by other techniques.

It is likely that many creative combinations of different ion mobility techniques, different types of spectroscopy, and different means of spectroscopic detection are yet to come.

\section{AUTHOR INFORMATION}

\section{Corresponding Author}

*E-mail: thomas.rizzo@epfl.ch.

ORCID $\odot$

Thomas R. Rizzo: 0000-0003-2796-905X

Present Address

${ }^{\dagger}$ TOFWERK AG, Uttigenstrasse 22, CH-3600 Thun, Switzerland.

Notes

The authors declare no competing financial interest.

\section{Biographies}

Michael Z. Kamrath earned his Ph.D. in chemistry from Yale University in 2012 and did postdoctoral research at the EPFL in Lausanne, Switzerland. His research interests drive him to develop novel instrumentation combining ion mobility, spectroscopy, and mass spectrometry. He currently leads ion mobility R\&D at Tofwerk A.G. in Switzerland.

Thomas R. Rizzo earned his Ph.D. in Chemistry from the University of Wisconsin-Madison in 1983 and did postdoctoral work at the University of Chicago. After eight years on the faculty of the University of Rochester, he was named Professor of Chemistry at the EPFL in Lausanne, Switzerland. His current research interests are at the intersection of molecular spectroscopy and mass spectrometry.

\section{ACKNOWLEDGMENTS}

We are grateful to the EPFL and the Swiss National Science Foundation (Grant Number 200020 165908) for the financial support of this work. We also thank Prof. David Clemmer for the loan of the ion mobility drift tube and associated electronics as well as for many helpful discussions.

\section{REFERENCES}

(1) Lanucara, F.; Holman, S. W.; Gray, C. J.; Eyers, C. E. The power of ion mobility-mass spectrometry for structural characterization and the study of conformational dynamics. Nat. Chem. 2014, 6, 281-294.

(2) Jurneczko, E.; Barran, P. E. How useful is ion mobility mass spectrometry for structural biology? The relationship between protein crystal structures and their collision cross sections in the gas phase. Analyst 2011, 136, 20-28.

(3) Bohrer, B. C.; Mererbloom, S. I.; Koeniger, S. L.; Hilderbrand, A. E.; Clemmer, D. E. Biomolecule Analysis by Ion Mobility Spectrometry. In Annual Review of Analytical Chemistry; Annual Reviews: Palo Alto, 2008; Vol. 1, pp 293-327.

(4) Voronina, L.; Masson, A.; Kamrath, M.; Schubert, F.; Clemmer, D.; Baldauf, C.; Rizzo, T. Conformations of Prolyl-Peptide Bonds in the Bradykinin 1-5 Fragment in Solution and in the Gas Phase. J. Am. Chem. Soc. 2016, 138, 9224-9233.
(5) Polfer, N. C.; Oomens, J. Vibrational Spectroscopy of Bare and Solvated Ionic Complexes of Biological Relevance. Mass Spectrom. Rev. 2009, 28, 468-494.

(6) Oomens, J.; Sartakov, B. G.; Meijer, G.; Von Helden, G. Gasphase infrared multiple photon dissociation spectroscopy of massselected molecular ions. Int. J. Mass Spectrom. 2006, 254, 1-19.

(7) Wolk, A. B.; Leavitt, C. M.; Garand, E.; Johnson, M. A. Cryogenic Ion Chemistry and Spectroscopy. Acc. Chem. Res. 2014, 47, 202-210.

(8) Koeniger, S. L.; Merenbloom, S. I.; Clemmer, D. E. Evidence for many resolvable structures within conformation types of electrosprayed ubiquitin ions. J. Phys. Chem. B 2006, 110, 7017-7021.

(9) Koeniger, S. L.; Merenbloom, S. I.; Valentine, S. J.; Jarrold, M. F.; Udseth, H. R.; Smith, R. D.; Clemmer, D. E. An IMS-IMS Analogue of MS-MS. Anal. Chem. 2006, 78, 4161-4174.

(10) Vonderach, M.; Ehrler, O. T.; Weis, P.; Kappes, M. M. Combining Ion Mobility Spectrometry, Mass Spectrometry, and Photoelectron Spectroscopy in a High-Transmission Instrument. Anal. Chem. 2011, 83, 1108-1115.

(11) Masson, A.; Kamrath, M. Z.; Perez, M. A. S.; Glover, M. S.; Rothlisberger, U.; Clemmer, D. E.; Rizzo, T. R. Infrared Spectroscopy of Mobility-Selected H+-Gly-Pro-Gly-Gly (GPGG). J. Am. Soc. Mass Spectrom. 2015, 26, 1444-1454.

(12) Masellis, C.; Khanal, N.; Kamrath, M. Z.; Clemmer, D. E.; Rizzo, T. R. Cryogenic Vibrational Spectroscopy Provides Unique Fingerprints for Glycan Identification. J. Am. Soc. Mass Spectrom. 2017, 28, 2217-2222.

(13) Kamrath, M. Z.; Relph, R. A.; Guasco, T. L.; Leavitt, C. M.; Johnson, M. A. Vibrational predissociation spectroscopy of the H-2tagged mono- and dicarboxylate anions of dodecanedioic acid. Int. J. Mass Spectrom. 2011, 300, 91-98.

(14) Kamrath, M. Z.; Garand, E.; Jordan, P. A.; Leavitt, C. M.; Wolk, A. B.; Van Stipdonk, M. J.; Miller, S. J.; Johnson, M. A. Vibrational Characterization of Simple Peptides Using Cryogenic Infrared Photodissociation of H-2-Tagged, Mass-Selected Ions. J. Am. Chem. Soc. 2011, 133, 6440-6448.

(15) Kamrath, M. Z.; Perez, M. A. S.; Glover, M. S.; Rothlisberger, U.; Clemmer, D. E.; Rizzo, T. R. Structural role of cis/trans proline isomerization in cabTRP Ia as determined by infrared spectroscopy of its mobility selected ions. Manuscript in preparation.

(16) Papadopoulos, G.; Svendsen, A.; Boyarkin, O. V.; Rizzo, T. R. Spectroscopy of mobility-selected biomolecular ions. Faraday Discuss. 2011, 150, 243-255.

(17) Barnett, D. A.; Belford, M.; Dunyach, J.-J.; Purves, R. W. Characterization of a Temperature-Controlled FAIMS System. J. Am. Soc. Mass Spectrom. 2007, 18, 1653-1663.

(18) Robinson, E. W.; Shvartsburg, A. A.; Tang, K.; Smith, R. D. Control of Ion Distortion in Field Asymmetric Waveform Ion Mobility Spectrometry via Variation of Dispersion Field and Gas Temperature. Anal. Chem. 2008, 80, 7508-7515.

(19) Aksenov, A. A.; Kapron, J. T. Behaviour of tetraalkylammonium ions in high-field asymmetric waveform ion mobility spectrometry. Rapid Commun. Mass Spectrom. 2010, 24, 1392-1396.

(20) Papadopoulos, G.; Svendsen, A.; Boyarkin, O. V.; Rizzo, T. R. Conformational Distribution of Bradykinin [bk+2 $\mathrm{H}](2+)$ Revealed by Cold Ion Spectroscopy Coupled with FAIMS. J. Am. Soc. Mass Spectrom. 2012, 23, 1173-1181.

(21) Pierson, N. A.; Chen, L.; Russell, D. H.; Clemmer, D. E. CisTrans Isomerizations of Proline Residues Are Key to Bradykinin Conformations. J. Am. Chem. Soc. 2013, 135, 3186-3192.

(22) Svendsen, A.; Rizzo, T. R. Conformer-selected vibrational spectroscopy of bradykinin $[\mathrm{M}+2 \mathrm{H}] 2+$. Unpublished work.

(23) Frankevich, V. E.; Barylyuk, K. V.; Martinez-Lozano Sinues, P.; Zenobi, R. Ion mobility spectrometry coupled to laser-induced fluorescence for probing the electronic structure and conformation of gas-phase ions. J. Anal. Chem. 2014, 69, 1215-1219.

(24) Frankevich, V.; Martinez-Lozano Sinues, P.; Barylyuk, K.; Zenobi, R. Ion Mobility Spectrometry Coupled to Laser-Induced Fluorescence. Anal. Chem. 2013, 85, 39-43. 
(25) Warnke, S.; Seo, J.; Boschmans, J.; Sobott, F.; Scrivens, J. H.; Bleiholder, C.; Bowers, M. T.; Gewinner, S.; Schöllkopf, W.; Pagel, K.; von Helden, G. Protomers of Benzocaine: Solvent and Permittivity Dependence. J. Am. Chem. Soc. 2015, 137, 4236-4242.

(26) Daly, S.; MacAleese, L.; Dugourd, P.; Chirot, F. Combining Structural Probes in the Gas Phase - Ion Mobility-Resolved ActionFRET. J. Am. Soc. Mass Spectrom. 2018, 29, 133-139.

(27) Voronina, L.; Scutelnic, V.; Masellis, C.; Rizzo, T. R. Can mutational Analysis be used to assist structure determination of peptides? J. Am. Chem. Soc. 2018, 140, 2401-2404.

(28) Adamson, B. D.; Coughlan, N. J. A.; Markworth, P. B.; Continetti, R. E.; Bieske, E. J. An ion mobility mass spectrometer for investigating photoisomerization and photodissociation of molecular ions. Rev. Sci. Instrum. 2014, 85, 123109.

(29) Adamson, B. D.; Coughlan, N. J. A.; da Silva, G.; Bieske, E. J. Photoisomerization Action Spectroscopy of the Carbocyanine Dye DTC+ in the Gas Phase. J. Phys. Chem. A 2013, 117, 13319-13325.

(30) Simon, A.-L.; Chirot, F.; Choi, C. M.; Clavier, C.; Barbaire, M.; Maurelli, J.; Dagany, X.; MacAleese, L.; Dugourd, P. Tandem ion mobility spectrometry coupled to laser excitation. Rev. Sci. Instrum. 2015, 86, 094101.

(31) Choi, C. M.; Simon, A.-L.; Chirot, F.; Kulesza, A.; Knight, G.; Daly, S.; MacAleese, L.; Antoine, R.; Dugourd, P. Charge, Color, and Conformation: Spectroscopy on Isomer-Selected Peptide Ions. J. Phys. Chem. B 2016, 120, 709-714.

(32) Scutelnic, V.; Perez, M. A. S.; Marianski, M.; Warnke, S.; Gregor, A.; Rothlisberger, U.; Bowers, M. T.; Baldauf, C.; Von Helden, G.; Rizzo, T. R.; Seo, J. The Structure of the Protonated Serine Octamer. J. Am. Chem. Soc. 2018, DOI: 10.1021/jacs.8b02118.

(33) Koeniger, S. L.; Merenbloom, S. I.; Sevugarajan, S.; Clemmer, D. E. Transfer of Structural Elements from Compact to Extended States in Unsolvated Ubiquitin. J. Am. Chem. Soc. 2006, 128, 1171311719.

(34) Pierson, N. A.; Valentine, S. J.; Clemmer, D. E. Evidence for a Quasi-Equilibrium Distribution of States for Bradykinin $[\mathrm{M}+3 \mathrm{H}](3+)$ Ions in the Gas Phase. J. Phys. Chem. B 2010, 114, 7777-7783.

(35) Silveira, J. A.; Fort, K. L.; Kim, D.; Servage, K. A.; Pierson, N. A.; Clemmer, D. E.; Russell, D. H. From Solution to the Gas Phase: Stepwise Dehydration and Kinetic Trapping of Substance P Reveals the Origin of Peptide Conformations. J. Am. Chem. Soc. 2013, 135, 19147-19153.

(36) Ruotolo, B. T.; Robinson, C. V. Aspects of native proteins are retained in vacuum. Curr. Opin. Chem. Biol. 2006, 10, 402-408.

(37) Badman, E. R.; Hoaglund-Hyzer, C. S.; Clemmer, D. E. Monitoring structural changes of proteins in an ion trap over similar to 10-200 ms: Unfolding transitions in cytochrome c ions. Anal. Chem. 2001, 73, 6000-6007.

(38) Fort, K. L.; Silveira, J. A.; Pierson, N. A.; Servage, K. A.; Clemmer, D. E.; Russell, D. H. From Solution to the Gas Phase: Factors That Influence Kinetic Trapping of Substance P in the Gas Phase. J. Phys. Chem. B 2014, 118, 14336-14344.

(39) Nagornova, N. S.; Guglielmi, M.; Doemer, M.; Tavernelli, I.; Rothlisberger, U.; Rizzo, T. R.; Boyarkin, O. V. Cold-Ion Spectroscopy Reveals the Intrinsic Structure of a Decapeptide. Angew. Chem., Int. Ed. 2011, 50, 5383-5386.

(40) Silveira, J. A.; Servage, K. A.; Gamage, C. M.; Russell, D. H. Cryogenic Ion Mobility-Mass Spectrometry Captures Hydrated Ions Produced During Electrospray Ionization. J. Phys. Chem. A 2013, 117, 953-961. 